MATEC Web of Conferences 1, 10008 (2012)

DOI: $10.1051 /$ matecconf $/ 20120110008$

(C) Owned by the authors, published by EDP Sciences, 2012

\title{
Liquefaction of Tangier soils by using physically based reliability analysis modelling
}

\author{
N. Touil ${ }^{1}$, A. Khamlichi ${ }^{2}$, A. Jabbouri ${ }^{1}$, P. Dubujet ${ }^{3}$ \\ ${ }^{1}$ Laboratoire MGC, FST Tanger, Morocco \\ ${ }^{2}$ Laboratoire MAS, FS Tétouan, Morocco \\ ${ }^{3}$ Université de Lyon, Lyon, France
}

\begin{abstract}
Approaches that are widely used to characterize propensity of soils to liquefaction are mainly of empirical type. The potential of liquefaction is assessed by using correlation formulas that are based on field tests such as the standard and the cone penetration tests. These correlations depend however on the site where they were derived. In order to adapt them to other sites where seismic case histories are not available, further investigation is required. In this work, a rigorous one-dimensional modelling of the soil dynamics yielding liquefaction phenomenon is considered. Field tests consisting of core sampling and cone penetration testing were performed. They provided the necessary data for numerical simulations performed by using DeepSoil software package. Using reliability analysis, the probability of liquefaction was estimated and the obtained results were used to adapt Juang method to the particular case of sandy soils located in Tangier.
\end{abstract}

Keywords: Correlation, liquefaction, probabilities, seism, soil

\section{Introduction}

Loose saturated granular soils could experience great deformations during the occurrence of severe earthquakes. These could have destructive effects and cause huge damage to buildings and infrastructures. Liquefaction is associated to the apparition of excessive local pore water pressures. Loss of resistance and rigidity emerge, in case of insufficiently compacted and undrained soils, as the applied shear stresses are transferred spontaneously from total stresses to pore water pressures [1]. Dangerous reduction of the effective stresses occurs then. For sandy soils having a loose granular curve, pore pressure could jump to sufficiently high values that could compensate the total confining pressure, and the soil undergoes great strains as its behaviour becomes of fluidic type.

In order to characterize vulnerability of soils to liquefaction phenomenon a lot of empirical methods have been developed. Most of these methods have been introduced by Seed [2]. They are based upon comparing results gathered form sites where earthquakes have occurred and examining among them those for which liquefaction had taken place. In these approaches, liquefaction potential is determined by correlating it to some given propriety of the considered soil which measures its capacity of resistance to liquefaction. The early semi-empirical methods for evaluating liquefaction potential belong to what it is called the stress approach. Among these approaches, Juang method [3] is very interesting as it was derived based on neural network modelling. Other approaches were also proposed such as that of Dobry [4] who has introduced the strain approach and that of Park et al. [5] who have introduced the energetic approach. A lot of other empirical methods are reported in the literature [6].

Liquefaction of soils could also be stated by means of rational approaches based on analytic methods where mechanical models of the soil behaviour are used. Among these approaches, the partially coupled formulation $[7,8]$ considers the soil dynamical equations of equilibrium with a nonlinear soil constitutive law under the presence of viscous damping. A phenomenological law describing generation of pore pressures as function of total stresses and strains under undrained conditions is proposed along with a diffusive mechanism describing dissipation of pore water pressures. This approach is different from the uncoupled formulation in that a nonlinear constitutive soil law is used with variable bulk and shear modules that are 
actualized at each step. Pore water pressure generation is also well described by means of a model using up to seven parameters that are identified from laboratory cyclic shear and consolidation tests. At each step, the pore pressure is used to compute the effective stress which is used then to actualize the soil elastic constants. A comparative study between coupled and partially coupled formulations has been conducted by Arulanandan et al. [9]. The conclusion was that the partially coupled approach is sufficient to analyse liquefaction by means of the simplified procedure as the obtained soil accelerations are conservative. This motivates using the partially coupled approach in order to assess analytically liquefaction potential of soils. It should be noticed that this approach needs only a limited number of experimental results in comparison with the complete coupled approach where a huge experimental work is necessary for identifying the soil behavior parameters; otherwise its accuracy is altered and its use would not be effective.

The objective of this work is to investigate to what extent the semi-empirical Juang method [3] can predict liquefaction for Tangier soils by comparing its predictions with the results of a physically based onedimensional modelling according the partially coupled formulation. This will be assessed in terms of probability of liquefaction as obtained by a proper reliability analysis. It will provide a way to adapt the empirical method of Juang for predicting liquefaction of Tangier soils. A case study is examined for this purpose.

\section{Materials and methods}

DeepSoil [10] is a one-dimensional site response analysis program that can perform both equivalent linear and nonlinear analyses in the framework of partially coupled formulation approach to soil liquefaction problem. As the soil is sandy, pore water pressure is assumed to be given by the law of Dorby et al. [4]. This phenomenological law predicts the inter-pore pressure $u_{N}$ to be given under the following form

$u_{N}=\frac{2 p N_{c} f F\left(\gamma_{c t}-\gamma_{t u p}\right)^{s}}{1+2 N_{c} f F\left(\gamma_{c t}-\gamma_{t u p}\right)^{s}}$

where $N_{c}$ is the number of cycles, $\gamma_{\text {tup }}$ the shear limit strain and $\gamma_{c t}$ the last known shear undergone before sign changing. Coefficient $\gamma_{\text {tup }}$ is comprised between $0.01 \%$ and $0.02 \%$ for most of sands. Parameters $f, p$ and $F$ enable adjusting the model to experimental results. Triaxial non drained cyclic tests are used for that. Because of the hydro-mechanical coupling taking place in the porous medium, the generated excess pore water pressure modifies the effective shear stress-strain behavior curve. This is assumed to happen according to the Matasovic model [11] as follows

$$
\tau_{N}=\frac{\sqrt{1-u_{N}} \bar{G}_{N} \gamma}{1+\beta\left(\frac{\sqrt{1-u_{N}} \bar{G}_{N}}{\left(1-u_{N}^{v}\right) \bar{\tau}_{N}}|\gamma|\right)^{s}}
$$

where $\bar{G}_{N}$ is the initial shear modulus, $\bar{\tau}_{N}$ the normalised shear strength and $\gamma$ the shear strain. Parameters $\beta, s$ and $v$ are material constants.

According to DeepSoil modelling, the soil is assumed to be a vertical column that is constituted by a given number of layers. Parameters for each layer are identified from laboratory and in situ tests. When the boundary conditions are specified and the water table level is entered, the seismic acceleration which reproduces a typical seismic motion is imposed at the soil substratum. Analysis of the liquefaction potential for a given depth is directly performed on the obtained results by examining the ratio of inter-pore pressure over the effective soil stress.

Chen and Juang [12] have proposed in 1999 to evaluate liquefaction propensity in terms of probabilities. They have introduced the probability of liquefaction $P_{L}$ defined as function of the safety factor $F_{s}$ under the following form

$$
P_{L}=\frac{1}{1+\left(\frac{F_{s}}{A}\right)^{B}}
$$

Juang et al. [3] have calculated the coefficients for the limit state $g\left(F_{s}\right)=0.83-F_{s}$ and have obtained $A=0.96$ and $B=4.5$.

In the present study, reliability analysis approach is applied to assess the probability of liquefaction on a rational basis $[13,14]$. An interpolation formula having the form of equation (3) is then derived to get directly the probability for the limit state defined as pore water pressure over effective vertical stress equal to $83 \%$.

\section{Results and discussion}

A campaign of tests was conducted in the site of Tangier City Centre. 16 core sampling tests and 18 CPT tests were performed, [15]. To achieve reliability analysis a design of experiment (DOE) full factorial table was used to derive surface response based models giving pore water pressure as function of four factors. These last include the dimensionless seismic surface acceleration $\alpha=a_{\max } / g$ where $g$ is the gravity acceleration, the seismic fundamental frequency $F$, the water table level $H$ and the shear modulus $G$. Three levels were selected for each factor. They were defined by choosing a mean value and by using the coefficient 0.8 and 1.2 to obtain the low and high levels. Table 1 gives the levels chosen for each factor at depth $z=7 \mathrm{~m}$. 


\begin{tabular}{|c|c|c|c|}
\hline & Low & Medium & High \\
\hline$\alpha\left(m . s^{-2}\right)$ & 4.63 & 5.79 & 6.94 \\
\hline$F(H z)$ & 1.343 & 1.679 & 2.0145 \\
\hline$H(m)$ & 2.74 & 4.24 & 5.74 \\
\hline$G(M P a)$ & 311.8 & 389.7 & 478.4 \\
\hline
\end{tabular}

Table 1: Levels of the factors used to derive RSM models

Simulations were performed for data corresponding to the site where the complex Tangier City Center was built [29], for which the following parameters were identified: damping was fixed to the value $0.5 \%, \beta=0.8$, $s=0.7, f=1, p=1.1, F=2.6, \gamma_{\text {tup }}=0.015 \%$ and $\mathrm{v}=3.8$.

DeepSoil performs the analysis and returns the results in terms of the acceleration, strain, shear stress over effective vertical stress versus time of strain, pore water pressure over effective vertical stress versus time, response spectra.

A total number of $4^{3}=81$ calculations were performed. The results giving pore water pressure over effective vertical stress (PWP) as function of time an depth were obtained. Figure 1 gives, for the combination corresponding to medium levels of factors, the reduced PWP as function of time for the three depths $z=5 \mathrm{~m}$, $z=7 \mathrm{~m}$ and $z=9 \mathrm{~m}$.

Using interpolation of the obtained results response surface models giving the reduced PWP can be derived. A total number of 9 RSM models were identified.

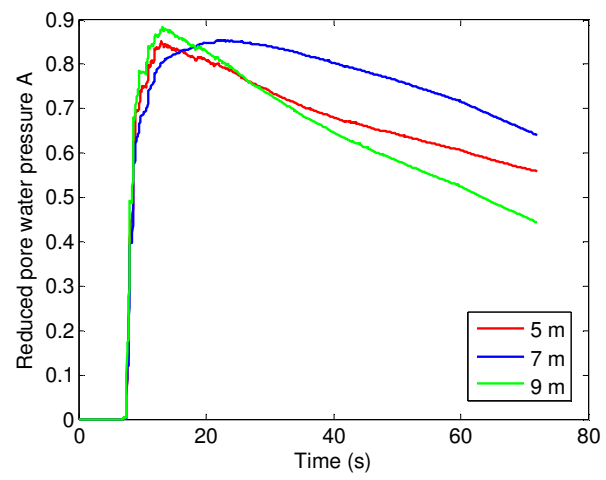

Fig.1. Reduced pore water pressure for $G_{\text {Medium }}, A_{\text {Medium }}$,

$$
F_{\text {Medium }} \text { and } H_{\text {Medium }}
$$

This enabled to express the liquefaction limit state for a given value of $G$ under the following

$$
g(A, F, H)=0.83-Q(A, F, H)
$$

Variables $\alpha, F, H$ are assumed to be random and normally distributed. Their means are the values defining each combination and their standard deviations are chosen to be given according to table 2 .

\begin{tabular}{|c|c|}
\hline & Standard deviation \\
\hline$\alpha$ & $10 \%$ \\
\hline$F$ & $10 \%$ \\
\hline$H$ & $17.5 \%$ \\
\hline
\end{tabular}

Table 2: Standard deviations of the random factors $\alpha$, $F$ and $H$

Predictions of probabilities provided by the Juang empirical approach and those obtained by simulation results obtained from physical modelling under DeepSoil and using reliability analysis are compared in the following.

Considering the case $G=G_{\text {Medium }}$, table 3 gives comparison of results due to DeepSoil reliability analysis based method and those of Juang method. One can notice that the results are quite different in case of depth $z=7 \mathrm{~m}$. This could be attributed to the constants $A$ and $B$ appearing in equation (3). We propose here to identify these constants by assuming that the probability of liquefaction is equal to the reliability based probability $P_{f}$. The obtained results are: $A=0.824$ and $B=3.06$.

\begin{tabular}{|c|c|c|}
\hline$z(m)$ & $P_{f}$ & $P_{L}$ \\
\hline 5 & 0.3315 & 0.5646 \\
\hline 7 & 0.2989 & 0.7332 \\
\hline 9 & 0.0243 & 0.0287 \\
\hline
\end{tabular}

Table 3: Probabilities of liquefaction for DeepSoil reliability based method $P_{f}$ and for Juang method $P_{L}$

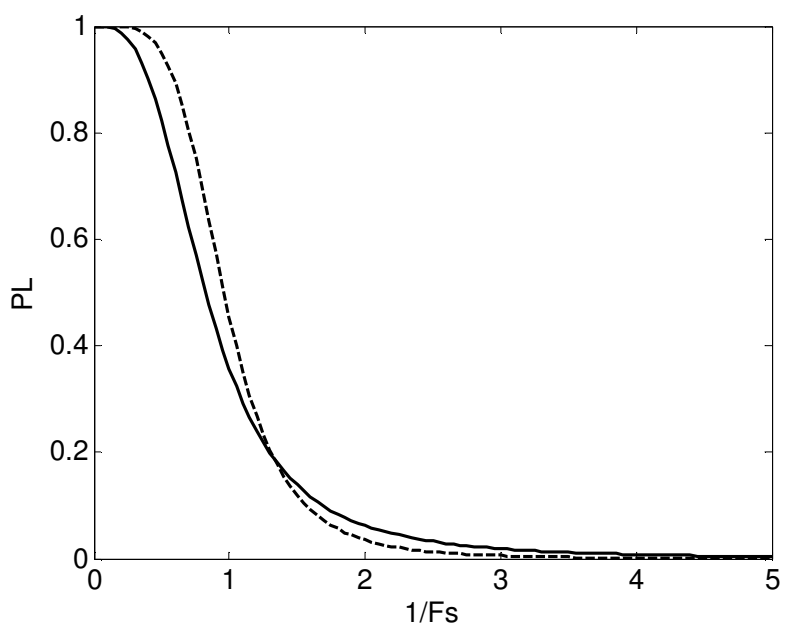

Fig.2. Comparison between Juang probability of liquefaction (discontinuous line) and modified probability of liquefaction (continuous line)

Figure 2 gives a comparison between probabilities obtained directly from equation (9) with the initial values 
of coefficients $A$ and $B$ (discontinuous line) and those computed with the new identified constants. One can notice that the original Juang method overestimates the probability of liquefaction in the useful domain $F s \geq 1$.

\section{Conclusion}

Physical modelling of liquefaction phenomenon was performed according to the partially coupled formulation by using DeepSoil software. Then, reliability analysis was achieved in order to calculate the probability of liquefaction associated to the limit state defined by pore water pressure over effective vertical stress greater than $83 \%$. In this analysis four factors were considered and three of them were assumed to be random variables that are normally distributed. These include shear modulus, seismic surface acceleration, seismic fundamental frequency and water table level. Comparison of predictions of probability of liquefaction as obtained by the empirical Juang method and the more accurate physical modelling was performed. The obtained results were found to be quite different. Assuming that the probability of liquefaction is given by the DeepSoil reliability analysis based method, a regression has enabled to modify the coefficients giving the probability of liquefaction according to Juang method. This work has shown how to use analytical modelling in order to correct the empirical Juang method with the objective to adapt it to the particular context of soils located in sites that are different from those used to derive it originally. Further verifications are needed to assess validity of the modified Juang probability of liquefaction that was proposed in this work.

\section{References}

1. Casagrande A. Liquefaction and cyclic deformation of sands - a critical review. In S.A.G. (Ed.), Fifth Panamerican Conference on Soil Mechanics and Foundation Engineering (pp. 79-133). Buenos Aires, Argentina: S.A.G., 1975

2. Seed B., Idriss I.M. Ground Motions and Soils Liquefaction During Earthquakes. Technical report, Earthquake Engineering Research Institute, 1982.

3. Juang C.H., Yuan H., Lee D.H., Lin P.S. Simplified cone penetration test-based method for evaluating liquefaction resistance of soils. J. Geotech. Geoenviron. Eng., 129(1), 66-80, 2003.

4. Dobry R. Soil properties and earthquake ground response, Volume 4, Proceedings of the Tenth European Conference on Soil Mechanics and Foundation Engineering, 1171-1187, 1994.

5. Park K., Kim S., Lee J., and Park I. Energy-based evaluation of excess pore pressure using damage potential. International Journal of Offshore and Polar Engineering, 2007.

6. Mitchell J.K. and Tseng D.J. Assessment of liquefaction potential by cone penetration resistance. Proc., H. Bolton Seed Memorial Symposium. Vol. 2. BiTech Pub., Richmond, British Columbia, May, pp. 335-350, 1990.
7. Lee M., Finn W. DESRA - Program for the Dynamic Effective Stress Response Analysis of Soil Deposites, Including Liquefaction Evaluation. Technical Report 36, Soil Mechanics Series. Departament of Civil Engineering, University of British Columbia, Vancouver, Canada, 1975.

8. Siddharthan R., Finn W. TARA2 - Two Dimensional Nonlinear, Static and Dynamic Response Analysis. Technical Report 74, Soil dynamics Group, University of British Columbia, Vancouver, Canada, 1982.

9. Arulanandan K., Muraleetharan K., Yogachandran C. Seismic response of soil deposits in San Fransisco marina distric. Journal of Geotechnical and Geoenvironmental Engineering, ASCE, 123, No 10, 965974, 1997.

10. Hashash Y.M.A, Groholski D.R., Phillips C. A., Park D. DEEPSOIL V3.7beta, User Manual and Tutorial. University of Illinois at Urbana-Champaign, USA, 88 p, 2009.

11. Matasovic N. and Vucetic M. Cyclic Characterization of Liquefiable Sands. ASCE Journal of Geotechnical and Geoenvironmental Engineering, 119(11):1805-1822, 1993.

12. Chen C.J., Juang C.H. Calibration of SPT- and CPT-based liquefaction evaluation methods. In: Mayne P., Hryciw R. (Eds.), Innovations Applications in Geotechnical Site Characterization. Geotechnical Special Publication, vol. 97. ASCE, Reston, VA, pp. 49- 64, 2000.

13. Iwasaki T., Arakawa T., Tokida K. Simplified procedures for assessing soil liquefaction during earthquakes. In Proceedings of the Conference on Soil Dynamics and Earthquake Engineering, Southampton, UK, Balkema, Rotterdam, the Netherlands. pp. 925-939, 1982.

14. Juang C.H., Ou C.Y., Lu C.C., Luo Z. Probabilistic framework for assessing liquefaction hazard at a given site in a specified exposure time using standard penetration testing. Can. Geotech. J. 47: 674-687, 2010 15. Touil N., Khamlichi A., Jabbouri A., Bezzazi M. Adaptation d'approches en vue d'évaluer le potentiel de liquéfaction des sols de Tanger. CFM'2009, Marseille, France, 2009. 